\title{
Noncommutative Phase Spaces by Coadjoint Orbits Method
}

\author{
Ancille NGENDAKUMANA ${ }^{\dagger}$, Joachim NZOTUNGICIMPAYE $\ddagger$ \\ and Leonard TODJIHOUNDE ${ }^{\dagger}$ \\ $\dagger$ Institut de Mathématiques et des Sciences Physiques, Porto-Novo, Benin \\ E-mail:nancille@yahoo.fr, leonardt@imsp-uac.org \\ $\ddagger$ Kigali Institute of Education, Kigali, Rwanda \\ E-mail: kimpaye@kie.ac.rw
}

Received May 24, 2011, in final form December 13, 2011; Published online December 18, 2011

http://dx.doi.org/10.3842/SIGMA.2011.116

\begin{abstract}
We introduce noncommutative phase spaces by minimal couplings (usual one, dual one and their mixing). We then realize some of them as coadjoint orbits of the anisotropic Newton-Hooke groups in two- and three-dimensional spaces. Through these constructions the positions and the momenta of the phase spaces do not commute due to the presence of a magnetic field and a dual magnetic field.
\end{abstract}

Key words: classical mechanics; noncommutative phase space; coadjoint orbit; symplectic realizations; magnetic and dual magnetic fields

2010 Mathematics Subject Classification: 22E60; 22E70; 37J15; 53D05; 53D17

\section{Introduction}

Noncommutative phase spaces provide mathematical backgrounds for the study of magnetic fields in physics. Noncommutativity appeared in nonrelativistic mechanics first in the work of Peierls [14] on the diamagnetism of conduction electrons. In relativistic quantum mechanics, noncommutativity was first examined in 1947 by Snyder [16]. During the last 15 years, noncommutative mechanics has been an important subject which attracted quite a lot of attention (see, e.g., [3, 4, 5, 6, 9, 19]).

Noncommutative phase space is defined as a space on which coordinates satisfy the commutation relations:

$$
\left\{q^{i}, q^{j}\right\}=G^{i j}, \quad\left\{q^{i}, p_{j}\right\}=\delta_{j}^{i}, \quad\left\{p_{i}, p_{j}\right\}=F_{i j},
$$

where $\delta_{j}^{i}$ is a unit matrix, whereas $G^{i j}$ and $F_{i j}$ are functions of positions and momenta. The physical dimensions of $G^{i j}$ and $F_{i j}$ are respectively $M^{-1} T$ and $M T^{-1}$, where $M$ represents a mass while $T$ represents a time.

The aim of this paper is to introduce the construction of noncommutative spaces by using different minimal couplings and the realization of some of them as coadjoint orbits $[10,11,17]$. The maximal coadjoint orbits of the anisotropic Newton-Hooke groups in two dimensions and in three dimensions are shown to be models of noncommutative spaces.

The paper is organized as follows. In Section 2 noncommutative phase spaces are introduced by generalizing the usual Hamiltonian equations to the cases where a magnetic field and a dual magnetic field are present. Section 3 is devoted to the study of planar mechanics in the following three situations:

- when a massive charged particle is in an electromagnetic field, 
- when a massless spring is in a dual magnetic field,

- when a pendulum is in an electromagnetic field and in a dual one.

It is shown in this paper that under the presence of these fields

- the massive charged particle acquires an oscillatory motion with a certain frequency,

- the massless spring acquires a mass,

- the pendulum appears like two synchronized oscillators.

The second and third results mentioned above are quite new. In Section 4 we construct, for the first time, the coadjoint orbits of the anisotropic Newton-Hooke groups in dimension two and dimension three. Then, we obtain noncommutative phase spaces in presence of a magnetic field and a dual magnetic field.

\section{Noncommutative phase spaces}

In this paragraph we recall Hamiltonian mechanics in both Darboux's coordinates (Section 2.1) and noncommutative coordinates (Section 2.2), the noncommutativity coming from the presence of two fields $F_{i j}$ and $G^{i j}$. We will distinguish three cases of noncommutative coordinates corresponding to the presence of the magnetic field only, of the dual magnetic field only and of the both fields simultaneously.

\subsection{Commutative coordinates}

It is known that a symplectic manifold is a $2 n$-dimensional manifold equipped with a closed nondegenerate 2 -form $\sigma$. If $\sigma_{a b}$ are the matrix elements of the matrix representing the symplectic form $\sigma$ and if $\sigma^{a b}$ are solutions of $\sigma_{b c} \sigma^{c a}=\delta_{b}^{a}$, then a Poisson bracket of two functions $f$ and $g$ belonging to $C^{\infty}(V, \mathbb{R})$ is given by

$$
\{f, g\}=\sigma^{a b} \frac{\partial f}{\partial z^{a}} \frac{\partial g}{\partial z^{b}} .
$$

The space $C^{\infty}(V, \mathbb{R})$ endowed with the Poisson bracket given by (1) is an infinite Lie algebra [1]. If $z^{a}=\left(p_{i}, q^{i}\right)$ are the canonical coordinates (Darboux's coordinates) on $V$, the symplectic form on $V$ is $\sigma=d p_{i} \wedge d q^{i}$, that means there is no coupling to a gauge field, and the Poisson bracket (1) becomes

$$
\{f, g\}=\frac{\partial f}{\partial p_{i}} \frac{\partial g}{\partial q^{i}}-\frac{\partial f}{\partial q^{i}} \frac{\partial g}{\partial p_{i}} .
$$

It follows that

$$
\left\{p_{k}, p_{i}\right\}=0, \quad\left\{p_{k}, q^{i}\right\}=\delta_{k}^{i}, \quad\left\{q^{k}, q^{i}\right\}=0 .
$$

That means the momenta $p_{i}$ as well as the positions $q^{i}$ are commutative.

It is also known that if $X_{f}$ is the Hamiltonian vector field associated to $f$, then $X_{f}(g)=\{f, g\}$ and the evolution equations under the flow $\Phi_{\exp \left(s X_{f}\right)}$ on $V$ generated by $X_{f}$ are

$$
\frac{d z^{a}}{d s}=X_{f}\left(z^{a}\right)
$$

which are exactly the usual Hamiltonian equations when $f$ is the energy.

Let us now introduce noncommutative coordinates by coupling the momentum $p_{i}$ with a magnetic potential $A_{i}$ and the position $q^{i}$ with a potential $A^{* i}$. 


\subsection{Noncommutative coordinates}

Let us consider the change of coordinates

$$
\pi_{i}=p_{i}-\frac{1}{2} F_{i k} q^{k}, \quad x^{i}=q^{i}-\frac{1}{2} p_{k} G^{k i} .
$$

The matrix form of (2) is

$$
\left(\begin{array}{c}
\pi_{i} \\
x^{i}
\end{array}\right)=\left(\begin{array}{cc}
\delta_{i}^{k} & -\frac{1}{2} F_{i k} \\
-\frac{1}{2} G^{i k} & \delta_{k}^{i}
\end{array}\right)\left(\begin{array}{l}
p_{k} \\
q^{k}
\end{array}\right)
$$

As

$$
\left(\begin{array}{cc}
\delta_{i}^{k} & -\frac{1}{2} F_{i k} \\
-\frac{1}{2} G^{i k} & \delta_{k}^{i}
\end{array}\right)=\left(\begin{array}{cc}
\delta_{i}^{j} & -\frac{1}{2} F_{i j} \\
0^{i j} & \delta_{j}^{i}
\end{array}\right)\left(\begin{array}{cc}
\delta_{j}^{s}-\frac{1}{4} F_{j m} G^{m s} & 0_{j s} \\
0^{j s} & \delta_{s}^{j}
\end{array}\right)\left(\begin{array}{cc}
\delta_{s}^{k} & 0^{s k} \\
-\frac{1}{2} G^{s k} & \delta_{k}^{s}
\end{array}\right),
$$

the transformation (2) is a change of coordinates if $\operatorname{det}\left(\delta_{j}^{s}-\frac{1}{4} F_{j m} G^{m s}\right) \neq 0$. It follows that [20, equation (5)]

$$
\left\{\pi_{i}, \pi_{k}\right\}=F_{i k}, \quad\left\{\pi_{i}, x^{k}\right\}=\delta_{i}^{k}, \quad\left\{x^{i}, x^{k}\right\}=G^{i k},
$$

i.e. the new momenta as well as the new configuration coordinates are noncommutative. The Jacobi identity implies that the $F_{i j}$ 's depend only on positions, that the $G^{i j}$ 's depend only on momenta and that the two 2-forms $\sigma_{1}=F_{i j}(x) d x^{i} \wedge d x^{j}$ and $\sigma_{2}=G^{i j}(\pi) d \pi_{i} \wedge d \pi_{j}$ are closed.

Let the Poisson brackets of two functions $f, g$ in the new coordinates be given by

$$
\{f, g\}_{\text {new }}=\frac{\partial f}{\partial \pi_{i}} \frac{\partial g}{\partial x^{i}}-\frac{\partial f}{\partial x^{i}} \frac{\partial g}{\partial \pi_{i}}=Y_{f}(g) .
$$

It follows that

$$
Y_{H}=X_{H}+G^{i j} \frac{\partial H}{\partial q^{i}} \frac{\partial}{\partial q^{j}}+F_{i j} \frac{\partial H}{\partial p_{i}} \frac{\partial}{\partial p_{j}} .
$$

The derivative of any function $f$ with respect to time $t$, in terms of $F$ and $G$, is then given by

$$
\frac{d f}{d t}=X_{H}(f)+G^{i j} \frac{\partial H}{\partial q^{i}} \frac{\partial f}{\partial q^{j}}+F_{i j} \frac{\partial H}{\partial p_{i}} \frac{\partial f}{\partial p_{j}},
$$

and the equations of motion are then given by

$$
\frac{d q^{k}}{d t}=\frac{\partial H}{\partial p_{k}}+G^{k i} \frac{\partial H}{\partial q^{i}}, \quad \frac{d p_{k}}{d t}=-\frac{\partial H}{\partial q^{k}}+F_{i k} \frac{\partial H}{\partial p_{i}} .
$$

If for example

$$
H=\frac{\delta^{i j} p_{i} p_{j}}{2 m}+V
$$

is the Hamiltonian with the potential energy $V$ depending only on the configuration coordinates $q^{i}$, the equations of motion are then

$$
\frac{d q^{k}}{d t}=\frac{p^{k}}{m}+G^{k i} \frac{\partial V}{\partial q^{i}}, \quad \frac{d p_{k}}{d t}=-\frac{\partial V}{\partial q^{k}}+F_{i k} \frac{p^{i}}{m} .
$$

They are equivalent to the modified Newton's second law $[5,15,20]$

$$
m \frac{d^{2} q^{k}}{d t^{2}}=-\frac{\partial V}{\partial q^{k}}+F_{i k} \frac{p^{i}}{m}+m G^{k i} \frac{d}{d t}\left(\frac{\partial V}{\partial q^{i}}\right) .
$$

This means that the noncommutativity of the momenta implies that the particle is accelerated and is not free even if the potential $V$ vanishes identically. 


\section{Couplings in planar mechanics}

In this section we construct explicitly these noncommutative phase spaces by introducing couplings. We start with the usual coupling of momentum with a magnetic potential. Then, we introduce the coupling of position with a dual potential and finish with a mixing model.

\subsection{Coupling of momentum with a magnetic field}

\subsubsection{Commutative coordinates}

Consider a four-dimensional phase space (a cotangent space to a plane) equipped with the Darboux's coordinates $\left(p_{i}, q^{i}\right)$. This means that the momenta as well as the positions commute. Consider also an electron with mass $m$ and an electric charge $e$, moving on a plane with the electromagnetic potential $A_{\mu}=\left(A_{i}=-\frac{1}{2} B \epsilon_{i k} q^{k}, \phi=E_{i} q^{i}\right)$ where a symmetric gauge has been chosen, $\vec{E}$ being an electric field while $\vec{B}$ is a magnetic field perpendicular to the plane. It is known that the dynamics of the particle is governed by the Hamiltonian

$$
H=\frac{\vec{p}^{2}}{2 m}-e \phi
$$

and that the equation of motion is

$$
m \frac{d^{2} \vec{q}}{d t^{2}}=e \vec{E}
$$

where the right hand side is the electric force.

\subsubsection{Noncommutative coordinates}

From the classical electromagnetism, it is known that the coupling of the momentum with the magnetic potential is given by the relations

$$
\pi_{i}=p_{i}+\frac{e B}{2} \epsilon_{i k} q^{k}, \quad x^{i}=q^{i} .
$$

The coordinates $\pi_{i}$ and $x^{i}$ are such that

$$
\left\{x^{i}, x^{k}\right\}=0, \quad\left\{\pi_{i}, x^{k}\right\}=\delta_{i}^{k}, \quad\left\{\pi_{i}, \pi_{k}\right\}=-e B \epsilon_{i k} .
$$

In the presence of an electromagnetic field, the momenta are noncommutative while the positions are commutative. Using the equation (3), we have

$$
F_{i j}=-e B \epsilon_{i j}, \quad G^{i j}=0^{i j} .
$$

Use of (5) and (7) into (4) gives rise the equations of motion

$$
m \frac{d^{2} \vec{q}}{d t^{2}}=e\left(\vec{E}+\frac{\vec{p}}{m} \times \vec{B}\right),
$$

where the right hand side represents the Lorentz force. Moreover, in noncommutative coordinates, the Hamiltonian (5) becomes

$$
H=\frac{\vec{\pi}^{2}}{2 m}-e \vec{E} \cdot \vec{x}+\frac{m \omega^{2} \vec{x}^{2}}{2}+\vec{\omega} \cdot \vec{L},
$$


where $\omega$ is the cyclotron frequency, $\vec{L}=\vec{x} \times \vec{p}$ is the orbital angular momentum and

$$
\vec{\omega}=\frac{e B}{2 m} \vec{n}
$$

with $\vec{n}$ the unit vector in the direction perpendicular to the plane. In the presence of a magnetic field, the massive particle has become an oscillator with frequency $\omega$ given above and then the equation of motion is

$$
m \frac{d^{2} \vec{x}}{d t^{2}}=e\left(\vec{E}+\frac{\vec{\pi}}{m} \times \vec{B}\right)
$$

where we recognize again the Lorentz force $\vec{f}_{\text {Lorentz }}=e \vec{E}+e \frac{\vec{\pi}}{m} \times \vec{B}$. Note that the relations (8) and (9) have the same form. The Newton's equations are then covariant under the coupling (6).

In the next two subsections, we present quite new theories associated with an unusual coupling of position with a dual magnetic field.

\subsection{Coupling of position with a dual field}

\subsubsection{Commutative coordinates}

Consider a massless spring with $k$ as a Hooke's constant and a dual charge $e^{*}$ in a dual magnetic field $B^{*}$. Suppose that the dynamics of the spring is governed by the Hamiltonian

$$
H=k \frac{\vec{q}^{2}}{2}-e^{*} \vec{p} \cdot \overrightarrow{E^{*}},
$$

where we have used the symmetric gauge. Moreover the analogue of the Newton's second equation is

$$
\frac{1}{k} \frac{d^{2} \vec{p}}{d t^{2}}=e^{*} \overrightarrow{E^{*}}
$$

where $\frac{d^{2} \vec{p}}{d t^{2}}$ is a yank, i.e. the second derivatives of momentum with respect to the time variable $t$, $e^{*} \overrightarrow{E^{*}}$ is a velocity while $e \vec{E}$ is a force as in the previous subsection.

\subsubsection{Noncommutative coordinates}

Let us consider the coupling of the position with the dual potential $A^{* i}$ depending on the momenta $p_{i}$

$$
\pi_{i}=p_{i}, \quad x^{i}=q^{i}+\frac{e^{*} B^{*}}{2} p_{k} \epsilon^{k i} .
$$

In this case, the Poisson brackets become

$$
\left\{x^{i}, x^{j}\right\}=-e^{*} B^{*} \epsilon^{i j}, \quad\left\{p_{k}, x^{i}\right\}=\delta_{k}^{i}, \quad\left\{p_{k}, p_{i}\right\}=0 .
$$

Therefore, in the presence of the dual field, positions do not commute while the momenta commute. Then

$$
F_{i j}=o_{i j}, \quad G^{i j}=-e^{*} B^{*} \epsilon^{i j} .
$$

Use (10) and (12) into (4) gives rise to the Newton's analogue equations are then

$$
\frac{1}{k} \frac{d^{2} \vec{p}}{d t^{2}}=e^{*} \overrightarrow{E^{*}}+e^{*} k \vec{q} \times \overrightarrow{B^{*}},
$$


the right hand side being a velocity. In noncommutative coordinates the Hamiltonian is

$$
H=\frac{k \vec{x}^{2}}{2}-e^{*} \vec{\pi} \cdot \vec{E}^{*}+\frac{\vec{\pi}^{2}}{2 m_{s}}-\vec{\omega} \cdot \vec{L}
$$

where the spring mass $m_{s}$ is defined by

$$
\frac{1}{m_{s}}=k \frac{e^{* 2} B^{* 2}}{4}
$$

while the vector $\vec{\omega}$ is given by

$$
\vec{\omega}=k \frac{e^{*} B^{*}}{2} \vec{n} .
$$

The Hooke's constant $k$ can be written as

$$
k=m_{s} \omega^{2} .
$$

In the presence of the dual field, the spring then acquires a mass $m_{s}$ and the equations of motion are given by

$$
\frac{1}{k} \frac{d^{2} \vec{\pi}}{d t^{2}}=e^{*} \overrightarrow{E^{*}}+e^{*} k \vec{x} \times \overrightarrow{B^{*}} .
$$

The vector $\overrightarrow{f^{*}}=e^{*}\left(\overrightarrow{E^{*}}+k \vec{x} \times \overrightarrow{B^{*}}\right)$ can be considered as a dual Lorentz force with the dimension of velocity. It represents for the spring what the Lorentz force represents for a charged particle. Here also the coupling (11) preserves the covariance of the Newton's analogue equations. Comparing (13) and (15) and using (14) into (15) we can conclude that $e^{*} \omega^{2}\left(\overrightarrow{E^{*}}+k \vec{x} \times \overrightarrow{B^{*}}\right)$ is a kind of jerk [13].

\subsubsection{Coupling with a magnetic field and with a dual field}

Now consider the case of a massive pendulum with mass $m$ and Hooke's constant $k$ under the action of an electromagnetic potential $A_{\mu}=\left(A_{i}, \phi\right)$ and a dual electromagnetic potential $A_{\mu}^{*}=\left(A_{i}^{*}, \phi^{*}\right)$ with $A_{i}=-\frac{1}{2} B \epsilon_{i k} q^{k}, \phi=E_{i} q^{i}, A_{i}^{*}=-\frac{1}{2} B^{*} p_{k} \epsilon_{k i}$ and $\phi^{*}=p_{i} E_{i}^{*}$, where $\vec{E}$ is an electric field and $\vec{E}^{*}$ its dual field while $\vec{B}$ is a magnetic field and $\vec{B}^{*}$ its dual field.

The corresponding motion is governed by the Hamiltonian

$$
H=\frac{\vec{p}^{2}}{2 m}+\frac{k \vec{q}^{2}}{2}-e \phi-e^{*} \phi^{*} .
$$

Let

$$
x^{i}=q^{i}+\frac{e^{*} B^{*}}{2} p_{k} \epsilon^{k i}, \quad \pi_{i}=p_{i}+\frac{e B}{2} \epsilon_{i k} q^{k}
$$

be the minimal coupling in the symmetric gauge; that is

$$
G^{i j}=-e^{*} B^{*} \epsilon^{i j}, \quad F_{i j}=-e B \epsilon_{i j} .
$$

We assume that the cyclotron frequency acquired by the massive charged particle is equal to the frequency of the massless spring:

$$
\frac{e B}{2}=m \omega, \quad \frac{e^{*} B^{*}}{2}=\frac{1}{m_{s} \omega},
$$


where $m_{s}$ is the acquired mass by the spring while

$$
\mu=\frac{m \cdot m_{s}}{m+m_{s}}
$$

is the reduced mass of the two synchronized massive oscillators. It follows that

$$
\left\{x^{i}, x^{j}\right\}=-e^{*} B^{*} \epsilon^{i j}, \quad\left\{\pi_{k}, x^{i}\right\}=\gamma \delta_{k}^{i}, \quad\left\{\pi_{k}, \pi_{i}\right\}=-e B \epsilon_{k i}
$$

with $\gamma=1+\frac{m}{m_{s}}$ and $m=\mu \gamma$.

In the presence of the two kind of field, the positions as well as the momenta do not commute. The Hamiltonian in noncommutative coordinates is written as

$$
H=\frac{\vec{\pi}^{2}}{2 \mu}+\frac{M \omega^{2} \vec{x}^{2}}{2}-e \phi-e^{*} \phi^{*}
$$

where $M=m+m_{s}$ is the total mass, $\phi=\vec{E} \cdot \vec{x}+\vec{n} \cdot \vec{E} \times \frac{\vec{\pi}}{m_{s} \omega}$ and $\phi^{*}=\vec{\pi} \cdot \overrightarrow{E^{*}}+\vec{n} \cdot m \omega \vec{x} \times \overrightarrow{E^{*}}$. Note that $M=m_{s} \gamma$.

The motion's equations in noncommutative coordinates are then

$$
\begin{aligned}
& \frac{d \vec{x}}{d t}=\frac{\vec{\pi}}{\mu}+e^{*}\left[\gamma \overrightarrow{E^{*}}+k \vec{x} \times \overrightarrow{B^{*}}-e \overrightarrow{B^{*}} \times \vec{E}\right], \\
& \frac{d \vec{\pi}}{d t}=-k \gamma \vec{x}+e\left[\gamma \vec{E}+\frac{\vec{\pi}}{m} \times \vec{B}-e^{*} \vec{B} \times \overrightarrow{E^{*}}\right],
\end{aligned}
$$

where the Hooke's constant $k$ is given by (14).

If the mass $m$ of the particle is very smaller than the mass $m_{s}$ acquired by the spring, i.e. $m \ll m_{s}$, then $\gamma$ becomes 1 and $\mu=m \ll M=m_{s}$. In that limit, the brackets (16) become

$$
\left\{x^{i}, x^{j}\right\}=-e^{*} B^{*} \epsilon^{i j}, \quad\left\{\pi_{k}, x^{i}\right\}=\delta_{k}^{i}, \quad\left\{\pi_{k}, \pi_{i}\right\}=-e B \epsilon_{k i},
$$

the Hamiltonian becomes

$$
H=\frac{\vec{\pi}^{2}}{2 m}+\frac{m_{s} \omega^{2} \vec{x}^{2}}{2}-e\left[\vec{E} \cdot \vec{x}+\vec{n} \cdot \vec{E} \times \frac{\vec{\pi}}{m_{s} \omega}\right]-e^{*}\left[\vec{\pi} \cdot \overrightarrow{E^{*}}+\vec{n} \cdot m \omega \vec{x} \times \overrightarrow{E^{*}}\right]
$$

and the equations of motion are given by

$$
\begin{aligned}
& \frac{d \vec{x}}{d t}=\frac{\vec{\pi}}{m}+e^{*}\left[\overrightarrow{E^{*}}+k \vec{x} \times \overrightarrow{B^{*}}-e \overrightarrow{B^{*}} \times \vec{E}\right], \\
& \frac{d \vec{\pi}}{d t}=-k \vec{x}+e\left[\vec{E}+\frac{\vec{\pi}}{m} \times \vec{B}-e^{*} \vec{B} \times \overrightarrow{E^{*}}\right] .
\end{aligned}
$$

The velocity $e e^{*} \overrightarrow{B^{*}} \times \vec{E}$ and the force $e e^{*} \overrightarrow{E^{*}} \times \vec{B}$ result from the coexistence of the two fields.

\section{Noncommutative phase spaces as coadjoint orbits of anisotropic Newton-Hooke groups}

It is well known that the dual of a Lie algebra has a natural Poisson structure whose symplectic leaves are the coadjoint orbits. These orbits will provide naturally noncommutative phase spaces.

In this section, we use orbit construction to realize noncommutative phase spaces on the anisotropic Newton-Hooke groups in two- and three-dimensional spaces, the anisotropic NewtonHooke groups $A N H_{ \pm}$being Newton-Hooke groups $N H_{ \pm}$without the rotation parameters [2]. Their Lie algebras have the structures

$$
\left[K_{i}, E\right]=P_{i}, \quad\left[P_{i}, E\right]= \pm \omega^{2} K_{i}, \quad i=1,2, \ldots, n,
$$


where

$$
\vec{K}=\frac{\partial}{\partial \vec{v}}, \quad \vec{P}=\frac{\partial}{\partial \vec{x}}, \quad E=\frac{\partial}{\partial t}+\vec{v} \cdot \frac{\partial}{\partial \vec{x}} \pm \omega^{2} \vec{x} \cdot \frac{\partial}{\partial \vec{v}} .
$$

Standard methods $[8,10,11,12]$ show that the structure of the central extensions of the Lie algebras $\mathcal{A N H}_{ \pm}$is

- in one-dimensional space

$$
[K, E]=P, \quad[P, E]= \pm \omega^{2} K, \quad[K, P]=M,
$$

- in two-dimensional spaces

$$
\begin{array}{ll}
{\left[K_{i}, K_{j}\right]=\frac{1}{c^{2}} J_{3} \epsilon_{i j}, \quad\left[K_{i}, E\right]=P_{i}, \quad\left[K_{i}, P_{j}\right]=M \delta_{i j},} \\
{\left[P_{i}, P_{j}\right]= \pm \frac{1}{r^{2}} J_{3} \epsilon_{i j},} & {\left[P_{i}, E\right]= \pm \omega^{2} K_{i},}
\end{array}
$$

- in three-dimensional spaces

$$
\begin{array}{ll}
{\left[K_{i}, K_{j}\right]=\frac{1}{c^{2}} J_{k} \epsilon_{i j}^{k}, \quad\left[K_{i}, E\right]=P_{i}, \quad\left[K_{i}, P_{j}\right]=M \delta_{i j},} \\
{\left[P_{i}, P_{j}\right]= \pm \frac{1}{r^{2}} J_{k} \epsilon_{i j}^{k},} & {\left[P_{i}, E\right]= \pm \omega^{2} K_{i}}
\end{array}
$$

where $r$ is a constant with the dimension of length, $c$ is a constant with the dimension of speed while $J_{k}$ is a rotation parameter around the $k^{\text {th }}$ axis.

\subsection{One-dimensional space case}

In this case $m$ is a trivial invariant. The other invariant, the solution of the Kirillov's system, is

$$
U=e-\frac{p^{2}}{2 m} \pm \frac{m \omega^{2} q^{2}}{2}
$$

where $q=\frac{k}{m}$. We denote the two-dimensional orbit by $\mathcal{O}_{(m, U)}$. It is not interesting for our study because there are one momentum and one position. Note that the symplectic realizations of $\mathrm{ANH}_{-}$and $\mathrm{ANH}_{+}$are respectively given by

$$
\begin{aligned}
L_{(v, x, t)}(p, q)= & (p \cos (\omega t)-m \omega q \sin (\omega t)-m v \cos (\omega t), \\
& \left.\frac{p}{m \omega} \sin (\omega t)+(q+x) \cos (\omega t)-\frac{v}{\omega} \sin (\omega t)\right)
\end{aligned}
$$

and

$$
\begin{gathered}
L_{(v, x, t)}(p, q)=(p \cosh (\omega t)+m \omega q \sinh (\omega t)-m(v \cosh (\omega t)-\omega x \sinh (\omega t)), \\
\left.\frac{p}{m \omega} \sinh (\omega t)+(q+x) \cosh (\omega t)-\frac{v}{\omega} \sinh (\omega t)\right) .
\end{gathered}
$$

Let $(p(t), q(t))=L_{(0,0, t)}(p, q)$, it follows that

$$
p(t)=p \cos (\omega t)-m \omega q \sin (\omega t), \quad q(t)=\frac{p}{m \omega} \sin (\omega t)+q \cos (\omega t)
$$


for $\mathrm{ANH}_{-}$and

$$
p(t)=p \cosh (\omega t)+m \omega q \sinh (\omega t), \quad q(t)=\frac{p}{m \omega} \sinh (\omega t)+q \cosh (\omega t)
$$

for $\mathrm{ANH}_{+}$. The equations of motion are then given by

$$
\frac{d p}{d t}= \pm m \omega^{2} q, \quad \frac{d q}{d t}=\frac{p}{m}
$$

for $A N H_{ \pm}$or equivalently $\frac{d^{2} q}{d t^{2}}= \pm \omega^{2} q$; which is a second order differential equation whose solutions are trigonometric functions for $\mathrm{ANH}_{-}$case and hyperbolic ones in $\mathrm{ANH}_{+}$case. It is for this reason that $A N H_{-}$describes a universe in oscillation while $A N H_{+}$describes a universe in expansion.

\subsection{Two-dimensional spaces case}

Let $m M^{*}+h J^{* 3}+k_{i} K^{* i}+p_{i} P^{* i}+e E^{*}$ be the general element of the dual of the central extended Lie algebra. Then $m$ and $h$ are trivial invariants under the coadjoint action of $A N H_{ \pm}$ in two-dimensional spaces. The other invariant, the solution of the Kirillov's system, is explicitly given by

$$
U=e-\frac{\vec{p}^{2}}{2 \mu_{e}} \pm \frac{\mu_{e} \omega^{2} \vec{q}^{2}}{2}
$$

with

$$
\mu_{e}=m \pm \frac{h}{\omega r^{2}}, \quad \vec{q}=\frac{\vec{k}}{\mu_{e}},
$$

where $h \omega_{0}=m c^{2}$ denotes the wave-particle duality, $\mu_{e}$ is an effective mass. The restriction of the Kirillov's matrix on the orbit is given by

$$
\Omega=\left(\begin{array}{cccc}
0 & \frac{h}{c^{2}} & m & 0 \\
-\frac{h}{c^{2}} & 0 & 0 & m \\
-m & 0 & 0 & \pm \frac{h}{r^{2}} \\
0 & -m & \mp \frac{h}{r^{2}} & 0
\end{array}\right) .
$$

By using relations (17), the duality wave-particle and the equality $c=\omega r$, we obtain that the Poisson brackets of two functions defined on the orbit are given by

$$
\{H, f\}=\frac{\partial H}{\partial p_{i}} \frac{\partial f}{\partial q^{i}}-\frac{\partial H}{\partial q^{i}} \frac{\partial f}{\partial p_{i}}+G^{i j} \frac{\partial H}{\partial q^{i}} \frac{\partial f}{\partial q^{j}}+F_{i j} \frac{\partial H}{\partial p_{i}} \frac{\partial f}{\partial p_{j}}, \quad i, j=1,2
$$

with

$$
G^{i j}=-\frac{\epsilon^{i j}}{m \omega_{0}}, \quad F_{i j}=-\left(m-\mu_{e}\right) \omega \epsilon_{i j}
$$

It follows that the magnetic field $B$ and its dual field $B^{*}$ are such that

$$
e^{*} B^{*}=\frac{1}{m \omega_{0}}, \quad e B=\left(m-\mu_{e}\right) \omega .
$$

The effective mass is then given in terms of the magnetic field as

$$
\mu_{e}=m-\frac{e B}{\omega} .
$$


The Hamilton's equations are then

$$
\frac{d \pi_{i}}{d t}=-\frac{\partial H}{\partial q^{i}} \pm\left(m-\mu_{e}\right) \omega \epsilon_{i k} \frac{\partial H}{\partial p_{k}}, \quad \frac{d x^{i}}{d t}=\frac{\partial H}{\partial p_{i}}+\frac{\epsilon^{i k}}{2 m \omega_{0}} \frac{\partial H}{\partial q^{k}} .
$$

The inverse of $\Omega$ is

$$
\Omega^{-1}=\left(\begin{array}{cccc}
0 & \pm \frac{\omega}{\mu_{e}} & -\frac{1}{\mu_{e}} & 0 \\
\mp \frac{\omega}{\mu_{e}} & 0 & 0 & -\frac{1}{\mu_{e}} \\
\frac{1}{\mu_{e}} & 0 & 0 & \frac{1}{\mu_{e} \omega_{0}} \\
0 & \frac{1}{\mu_{e}} & -\frac{1}{\mu_{e} \omega_{0}} & 0
\end{array}\right),
$$

where we have used the wave-particle duality and (17). Finally the orbit is equipped with the symplectic form

$$
\sigma=d p_{i} \wedge d q^{i}+\frac{1}{\mu_{e} \omega_{0}} \epsilon^{i j} d p_{i} \wedge d p_{j} \pm \mu_{e} \omega \epsilon_{i j} d q^{i} \wedge d q^{j}
$$

We observe that with the anisotropic Newton-Hooke group in two-dimensional spaces, the obtained phase spaces are completely noncommutative while the phase space obtained with the Galilei group in [3] is only partially noncommutative. This is due to the difference in the structure of their extended Lie algebras. In the Newton-Hooke case space translations as well pure Newton-Hooke transformations do not commute while only pure Galilei transformations do not commute in the Galilei case. Note that the nontrivial Lie brackets of the extended NewtonHooke Lie algebra in two-dimensional space are given by

$$
\begin{aligned}
& {\left[J, K_{i}\right]=K_{j} \epsilon_{i}^{j}, \quad\left[J, P_{i}\right]=P_{j} \epsilon_{i}^{j},} \\
& {\left[K_{i}, P_{j}\right]=M \delta_{i j}, \quad\left[K_{i}, E\right]=P_{i}, \quad\left[P_{i}, E\right]=\omega^{2} K_{i},}
\end{aligned}
$$

which means that the generators of space translations as well as pure Newton-Hooke transformations commute. One can not then associate a noncommutative phase space to the NewtonHooke group. It is then the absence of the symmetry rotations (anisotropy of the plane) which guaranties the noncommutative phase space for the anisotropic Newton-Hooke group.

\subsection{Three-dimensional spaces case}

Let $m M^{*}+h_{i} J^{* i}+k_{i} K^{* i}+p_{i} P^{* i}+e E^{*}, i=1,2,3$ be the general element of the dual of the central extended Lie algebra. Then $m$ and $h_{i}$ are trivial invariants under the coadjoint action of $A N H_{ \pm}$in three-dimensional spaces. We need another invariant. As we can verify, the Kirillov's form, in the basis $\left(K_{i}, P_{i}, E\right)$, is given by

$$
B_{\alpha \beta}=\left(\begin{array}{ccc}
\frac{h_{k} \epsilon_{i j}^{k}}{c^{2}} & m \delta_{i j} & p_{i} \\
-m \delta_{i j} & \pm \frac{h_{k} \epsilon_{i j}^{k}}{r^{2}} & \pm \omega^{2} k_{i} \\
p_{j} & \mp \omega^{2} k_{j} & 0
\end{array}\right) .
$$

The other invariant which is a solution of the Kirillov's system is

$$
U=e-\frac{p_{i} p_{j}\left(\Phi_{ \pm}^{-1}\right)^{i j}}{2 m}-\frac{m \omega^{2} q^{i} q^{j}\left(\Phi_{ \pm}^{-1}\right)_{i j}}{2}+\omega^{2} p_{i} q^{j}\left(\Phi_{ \pm}^{-1} A\right)_{j}^{i}
$$

where

$$
A_{i j}=\frac{h_{k} \epsilon_{i j}^{k}}{m c^{2}}, \quad \Phi_{ \pm}=I \pm \omega^{2} A \quad \text { and } \quad q_{i}=\frac{k_{i}}{m} .
$$


We see that $\Phi_{ \pm}$is a metric for $\mathbb{R}^{3}$. Let us denote the maximal coadjoint orbit by $O_{(m, \vec{h}, U)}$. The restriction of the Kirillov's form on the orbit is then

$$
\Omega=m\left(\begin{array}{cc}
A_{i j} & \delta_{i}^{j} \\
-\delta_{j}^{i} & \pm \omega^{2} A_{i j}
\end{array}\right)
$$

and its inverse is

$$
\Omega^{-1}=\frac{1}{m}\left(\begin{array}{cc} 
\pm \omega^{2}\left(A \Phi_{ \pm}^{-1}\right)_{i j} & \left(\Phi_{ \pm}^{-1}\right)_{i}^{j} \\
-\left(\Phi_{ \pm}^{-1}\right)_{j}^{i} & \left(A \Phi_{ \pm}^{-1}\right)^{i j}
\end{array}\right) .
$$

The maximal orbit is then equipped with the symplectic structure

$$
\sigma=\left(\Phi_{ \pm}^{-1}\right)_{j}^{i} d p_{i} \wedge d q^{j}+\frac{1}{m}\left(A \Phi_{ \pm}^{-1}\right)^{i j} d p_{i} \wedge d p_{j} \pm m \omega^{2}\left(A \Phi_{ \pm}^{-1}\right)_{i j} d q^{i} \wedge d q^{j}
$$

and it follows that the Poisson brackets of two functions defined on the orbit is then

$$
\{f, g\}=\left(\Phi_{ \pm}^{-1}\right)_{i}^{j}\left(\frac{\partial f}{\partial p_{i}} \frac{\partial g}{\partial q^{j}}-\frac{\partial f}{\partial q^{i}} \frac{\partial g}{\partial p_{j}}\right)+F_{i j} \frac{\partial f}{\partial p_{i}} \frac{\partial g}{\partial p_{j}}+G^{i j} \frac{\partial f}{\partial q^{i}} \frac{\partial g}{\partial q^{j}} .
$$

This implies that

$$
\left\{p_{i}, p_{j}\right\}=F_{i j}, \quad\left\{p_{i}, q^{j}\right\}=\left(\Phi_{ \pm}^{-1}\right)_{i}^{j}, \quad\left\{q^{i}, q^{j}\right\}=G^{i j}
$$

where the magnetic field $F_{i j}$ and the dual magnetic field $G^{i j}$ are given by

$$
F_{i j}= \pm m \omega^{2}\left(A \Phi_{ \pm}^{-1}\right)_{i j} \quad \text { and } \quad G^{i j}=\frac{1}{m}\left(A \Phi_{ \pm}^{-1}\right)^{i j} .
$$

Moreover the Hamilton's equations are

$$
\begin{aligned}
\frac{d p_{k}}{d t} & =-\left(\Phi_{ \pm}^{-1}\right)_{k}^{i} \frac{\partial H}{\partial q^{i}} \pm m \omega^{2}\left(A \Phi_{ \pm}^{-1}\right)_{i k} \frac{\partial H}{\partial p_{i}} \\
\frac{d q^{k}}{d t} & =\left(\Phi_{ \pm}^{-1}\right)_{i}^{k} \frac{\partial H}{\partial p_{i}}+\frac{1}{m}\left(A \Phi_{ \pm}^{-1}\right)^{i k} \frac{\partial H}{\partial q^{i}}
\end{aligned}
$$

With the anisotropic Newton-Hooke groups $A N H_{ \pm}$in three-dimensional spaces, we also have realized phase spaces where the momenta as well as the positions do not commute.

\section{Conclusion}

We know that we can introduce the classical electromagnetic interaction through the modified symplectic form $\sigma=d p_{i} \wedge d q^{i}+\frac{1}{2} F_{i j} d q^{i} \wedge d q^{j}[1,7,17]$. This has been initiated by J.M. Souriau [17] in the seventies. Recently many authors (see, e.g., $[3,4,18,19])$ generalized this modification of the symplectic form by introducing the so-called dual magnetic field such that $\sigma=d p_{i} \wedge d q^{i}+$ $\frac{1}{2} F_{i j} d q^{i} \wedge d q^{j}+\frac{1}{2} G^{i j} d p_{i} \wedge d p_{j}$. The fields $F$ and $G$ are responsible of the noncommutativity respectively of momenta and positions. In our paper we have introduced these fields firstly by minimal coupling momenta with magnetic potentials (the usual one), secondly by minimal coupling of positions with dual potentials and lastly by mixing the two couplings. We have also realized phase spaces endowed with modified symplectic structures as coadjoint orbits of the anisotropic Newton-Hooke groups in two and three-dimensional spaces. In all these cases, the fields are constant because they are coming from central extensions of Lie algebras. 


\section{References}

[1] Abraham R., Marsden J.E., Foundations of mechanics, 2nd ed., Benjamin/Cummings Publishing Co., Inc., Advanced Book Program, Reading, Mass., 1978.

[2] Derome J.R., Dubois J.G., Hooke's symmetries and nonrelativistic cosmological kinematics. I, Nuovo Cimento B 9 (1972), 351-376.

[3] Duval C., Horváthy P.A., The exotic Galilei group and the "Peierls substitution", Phys. Lett. B 479 (2000), 284-290, hep-th/0002233.

[4] Duval C., Horváthy P.A., Exotic Galilean symmetry in the non-commutative plane and the Hall effect, J. Phys. A: Math. Gen. 34 (2001), 10097-10107, hep-th/0106089.

[5] Duval C., Horváth Z., Horváthy P.A., Exotic plasma as classical Hall liquid, Internat. J. Modern Phys. B 15 (2001), 3397-3408, cond-mat/0101449.

[6] Grigore D.R., Transitive symplectic manifolds in $1+2$ dimensions, J. Math. Phys. 37 (1996), 240-253.

[7] Guillemin V., Sternberg S., Symplectic techniques in physics, Cambridge University Press, Cambridge, 1984.

[8] Hamermesh M., Group theory and its applications to physical problems, Addison-Wesley Series in Physics, Addison-Wesley Publishing Co., Inc., Reading, Mass. - London, 1962.

[9] Horváthy P.A., The non-commutative Landau problem, Ann. Physics 299 (2002), 128-140, hep-th/0201007.

[10] Kirillov A.A., Elements of theory of representations, Grundlehren der Mathematischen Wissenschaften, Band 220, Springer-Verlag, Berlin - New York, 1976.

[11] Kostant B., Quantization and unitary representations. I. Prequantization, in Lectures in Modern Analysis and Applications, III, Lecture Notes in Math., Vol. 170, Springer, Berlin, 1970, 87-208.

[12] Nzotungicimpaye J., Galilei-Newton law by group theoretical methods, Lett. Math. Phys. 15 (1988), 101110.

[13] Nzotungicimpaye J., Jerk by group theoretical methods, J. Phys. A: Math. Gen. 27 (1994), 4519-4526.

[14] Peierls R., On the theory of diamagnetism of conduction electrons, Z. Phys. 80 (1933), 763-791.

[15] Romero J.M., Santiago J.A., Vergara J.D., Newton's second law in a non-commutative space, Phys. Lett. A 310 (2003), 9-12, hep-th/0211165.

[16] Snyder H.S., Quantized space-time, Phys. Rev. 71 (1947), 38-41.

[17] Souriau J.-M., Structure des systèmes dynamiques, Maîtrises de Mathématiques Dunod, Paris, 1970.

[18] Vanhecke F.J., Sigaud C., da Silva A.R., Noncommutative configuration space. Classical and quantum mechanical aspects, Braz. J. Phys. 36 (2006), 194-207, math-ph/0502003.

[19] Vanhecke F.J., Sigaud C., da Silva A.R., Modified symplectic structures in cotangent bundles of Lie groups aspects, Braz. J. Phys. 39 (2009), 18-24, arXiv:0804.1251.

[20] Wei G.-F., Long C.-Y., Long Z.-W., Qin S.-J., Fu Q., Classical mechanics in non-commutative phase space, Chinese Phys. C 32 (2008), 338-341. 